\title{
Composición nutricional, consumo e índices de palatabilidad relativa de los frutos de tres acacias en la alimentación de ovejas y cabras
}

\author{
Intake, nutritional composition and index relative palatability of fruits three \\ acacias in feeding sheep and goats
F Quiroz-Cardoso ${ }^{a}$, S Rojas-Hernández ${ }^{b^{*}}$, J Olivares-Pérez ${ }^{b^{*}}$, E Hernández-Castro $^{\mathrm{a}}, \mathbf{R}$ Jiménez-Guillén ${ }^{\mathrm{c}}$, A Córdova-Izquierdo ${ }^{\mathrm{d}}, \mathbf{A}$, Villa-Mancera ${ }^{\mathrm{e}}, \mathbf{S}$ Abdel-Fattah $^{\mathrm{f}}$ \\ ${ }^{a}$ Maestría en Sistemas de Producción Agropecuaria, Universidad Autónoma de Guerrero, México \\ bUnidad Académica de Medicina Veterinaria y Zootecnia, Universidad Autónoma de Guerrero, Guerrero, México. \\ 'Instituto Nacional de Investigaciones Forestales Agrícolas y Pecuarias, Iguala, Guerrero, Guerrero, México. \\ dDepartamento de Producción Agrícola y Animal, Universidad Autónoma Metropolitana, Xochimilco, México. \\ eFacultad de Medicina Veterinaria y Zootecnia, Benemérita Universidad Autónoma de Puebla, Puebla, México.

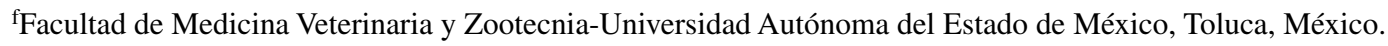

\section{SUMMARY}

\begin{abstract}
The aim of this study was to determine the nutritional composition of Acacia farnesiana, Acacia macilenta and Acacia cochliacantha fruits and determine the intake (FI) the relative palatability index (PI) and its correlation with the nutritional composition of fruit in sheep and goats. Sixteen females of sheep and goats were used: eight sheep (F1 Dorper X Pelibuey body weight $18.5 \pm 1.5 \mathrm{~kg}$ ) and eight Creole goats (body weight $20.6 \pm 1.8 \mathrm{~kg}$ ). Animals were housed in individual pens $(1.0 \mathrm{X} 1.20 \mathrm{~m}$ ) and fed on fruits ( $a d$ libitum) of the three acacias and corn stover (CS) for 24 hours during 15 days of experiment. Neutral and acid detergent fiber content, total phenols (TP) and condensed tannins $(\mathrm{CT})$ were different $(\mathrm{P}<0.01)$ between the fruits species. Fruits of A. farnesiana and $A$. cochliacantha had the highest $(\mathrm{P}<0.01)$ intake by goats, and sheep consumed more fruits of $A$. cochliacantha. On average, the FI was increased in goats $(\mathrm{P}<0.05)$, but the IP was increased in sheep $(\mathrm{P}<0.01)$. The FI and IP were positively correlated with protein content $(\mathrm{P}<0.01)$ and $\mathrm{TP}(\mathrm{P}<0.05)$ and negatively with the content of acid and neutral detergents fibers $(\mathrm{P}<0.01)$. It could be concluded that the fruits of A. cochliacantha and A. farnesiana have more nutritional value for sheep and goats because of their lower content of neutral and acid detergent fiber and highest palatability index observed.
\end{abstract}

Key words: intake, palatability, acacias, ruminants.

\section{RESUMEN}

El objetivo fue determinar la composición nutricional de los frutos de Acacia farnesiana, Acacia macilenta y Acacia cochliacantha y medir en las cabras y las ovejas el consumo de frutos (CF), el índice de palatabilidad relativo (IP) y su correlación con la composición nutricional de los frutos. Se utilizaron 16 hembras: 8 ovejas F1 Dorper X Pelibuey de 18,5 $\pm 1,5 \mathrm{~kg}$ de peso vivo y 8 cabras Criollas de 20,6 $\pm 1,8 \mathrm{~kg}$ de peso vivo. Los animales fueron alojados en corrales individuales $(1,0 \times 1,20 \mathrm{~m})$ y se les ofreció a libre acceso los frutos de las tres acacias y rastrojo de maíz (RM) al mismo tiempo las 24 horas durante 15 días. El contenido de fibras detergentes ácida y neutra $(\mathrm{P}<0,01)$, fenoles totales (FT) y taninos condensados (TC) $(\mathrm{P}<0,01)$ fue diferente entre los frutos. Los frutos de A. farnesiana y A. cochliacantha fueron los más consumidos por las cabras $(\mathrm{P}<0,01)$, y las ovejas consumieron más frutos de $A$. cochliacantha. En promedio el CF fue mayor en las cabras $(\mathrm{P}<0,05)$, pero el IP fue mayor en las ovejas $(\mathrm{P}<0,01)$. El CF y el IP tuvieron correlación positiva con el contenido de proteína $(\mathrm{P}<0,01)$ y FT $(\mathrm{P}<0,05)$ y negativa con el contenido de fibras detergentes ácida y neutra $(\mathrm{P}<0,01)$. Se concluye que los frutos de A. farnesiana y A. cochliacantha tienen más valor alimenticio para ovejas y cabras por el menor contenido de fibras detergentes neutro y ácido y el mayor índice de palatabilidad observado.

Palabras clave: consumo, palatabilidad, acacias, rumiantes.

\section{INTRODUCCIÓN}

La producción animal en las regiones tropicales se basa en el aprovechamiento de forrajes nativos o introducidos como principal fuente de nutrientes para los rumiantes, pero las fluctuaciones en la disponibilidad de las pasturas

Aceptado: 26.06.2014.

* Apartado Postal 12, Cd. Altamirano, Guerrero, México; saulrh@hotmail.com en la época seca obligan al uso de diversos recursos como las pajas remanentes de las cosechas de maíz y los frutos de diversos árboles para la alimentación de pequeños rumiantes (Rodríguez y col 2009, Rojas y col 2012, Vázquez y col 2012). En México la diversidad de árboles forrajeros es enorme y pueden contribuir al desarrollo integral de la ganadería por la diversidad de usos que aportan (Álvarez y col 2003, Sosa y col 2004, Olivares y col 2011). Las especies arbóreas del género acacia son muy abundantes y su follaje y frutos pueden ser valiosos para el aporte 
de energía y proteína en la dieta de pequeños rumiantes (Osuga y col 2008, García y col 2009). Sin embargo, se requiere de información acerca de su valor nutritivo y pruebas de consumo en animales para su uso adecuado en la alimentación de rumiantes (Mlambo y col 2008). Esta necesidad se acentúa si consideramos que la biomasa de árboles puede contener cantidades representativas de compuestos fenólicos y llegar a reducir el consumo y la digestibilidad de la dieta, y disminuir la producción animal (Ngwa y col 2003, Dey y col 2008).

Estudios basados en la observación directa de la alimentación y la ingesta, han sido adecuados para determinar el consumo y los índices relativos de palatabilidad en rumiantes alimentados con biomasa de árboles forrajeros (Kaitho y col 1996, Smith y col 2005, Rahim y col 2013). Olivares y col (2013) determinaron comparativamente que el fruto de Acacia cochliacantha fue preferido por las cabras con relación al fruto de Phitecellobium dulce; también Mokoboki y col (2011) reportaron diferencia en la preferencia de ovejas y cabras sobre el consumo de los frutos de cinco acacias en experimentos de palatabilidad. En relación con la especie animal Khan y Hussain (2012) reportan que las cabras presentan mayor consumo de biomasa de árboles que las ovejas. Kalio y col (2006) reportan que las variaciones en la preferencia de pequeños rumiantes sobre el consumo de los frutos de los árboles puede ser influido por el estado de madurez, procesamiento, la composición química, los compuestos fenólicos y por la especie o género arbóreo. En este estudio el objetivo fue evaluar el valor nutritivo, el consumo de frutos (CF) y el índice de palatabilidad relativo (IP) de los frutos de tres acacias en el trópico mexicano ofrecido a ovejas y cabras.

\section{MATERIAL Y MÉTODOS}

\section{LOCALIZACIÓN DEL ESTUDIO}

El estudio se realizó en la comunidad de Los Limones, en el municipio de Pungarabato, localizado en la región de la Tierra Caliente del estado de Guerrero ( $18^{\circ} 20^{\prime} 30^{\prime \prime}$ de latitud Norte y a $100^{\circ} 39^{\prime} 18^{\prime \prime}$ de longitud Oeste). El clima predominante es $\mathrm{Aw}_{0}$. La temperatura promedio es $28{ }^{\circ} \mathrm{C}$ y máxima de $46^{\circ} \mathrm{C}$. Una altura de $250 \mathrm{msnm}$ con una precipitación media anual de $750 \mathrm{~mm}$ (Fragoso 1990).

\section{RECOLECCIÓN DEL FRUTO}

Los frutos maduros de Acacia farnesiana (Huizache), Acacia cochliacantha (Cubata) y Acacia macilenta (Cuindera) fueron recolectados manualmente de los árboles en las aéreas de pastoreo, en el período de febrero a marzo de 2012. El rastrojo de maíz (RM) fue cosechado en la unidad productiva de la Unidad Académica de Medicina Veterinaria y Zootecnia de la Universidad Autónoma de Guerrero.
ANÁLISIS QUÍMICOS DE LOS FRUTOS Y DEL RASTROJO

DE MAÍZ

Se utilizaron tres muestras de $250 \mathrm{~g}$ de RM y de los frutos para los análisis químicos, se secaron a $60^{\circ} \mathrm{C}$ en una estufa de aire forzado hasta obtener peso constante y se molieron en un molino Willey con criba $\mathrm{N}^{\mathrm{o}} 1$, cada muestra se analizó por triplicado para el contenido de proteína cruda (PC), materia seca (MS), materia orgánica (MO), extracto etéreo (EE) con los métodos descritos por la AOAC (2000). La fibra detergente neutro (FDN) y fibra detergente ácida (FDA) se determinaron por el método de Van Soest y col (1991). Para la determinación de los fenoles totales y taninos condensados se tomaron muestras de $250 \mathrm{~g}$ de los frutos secos, se secaron a la sombra y molieron en un molino tipo Willey con criba de $1 \mathrm{~mm}$. El contenido de fenoles totales (FT) se determinó con Folin Ciocalteu y los taninos condensados (TC) (butanol-HCl) con el método descrito por Waterman y Mole (1994) (cuadro 1).

\section{MANEJO DE ANIMALES}

Se utilizaron 8 ovejas (peso vivo: $18,5 \pm 1,5 \mathrm{~kg}$ ) de siete meses de edad de la cruza Dorper-Pelibuey y 8 cabras (peso vivo: $20,6 \pm 1,8 \mathrm{~kg}$ ) criollas de siete meses de edad. Los animales fueron desparasitados vía oral con albendazole ${ }^{1}$ a razón de $1,5 \mathrm{~mL}$ por cada $10 \mathrm{~kg}$ de peso vivo (PV) y se les aplicó vía intramuscular vitaminas $\mathrm{ADE}^{2}$, a razón de 3 $\mathrm{mL}$ por animal. Fueron alojadas en corrales individuales de 1,0 x 1,20 m con libre acceso a un bebedero y a cuatro comederos donde se ofrecieron por separado $500 \mathrm{~g}$ de MS de cada uno de los tres frutos enteros y la dieta base (rastrojo de maíz molido) simultáneamente (cuadro 1). El orden de colocación de los frutos en los comederos se asignó al azar todos los días para evitar el reflejo de la costumbre. El agua se proporcionó ad libitum.

\section{VARIABLES EVALUADAS}

El experimento duró 25 días, 10 días de adaptación al confinamiento y 15 días de evaluación. El alimento ofrecido y el rechazado fueron pesados y registrados diariamente por cada animal. Con estos datos se calcularon los siguientes parámetros: $\mathrm{T} 1$ = ingesta diaria de RM, $\mathrm{Ti}=$ consumo promedio de los frutos donde $\mathrm{i}=1,2$ y 3 representó los frutos de los tres árboles (A. farnesiana, $A$. cochliacantha y A. macilenta) A1 = la cantidad de RM ofrecido, $\mathrm{Ai}=$ cantidad de fruto ofrecido de cada árbol.

El índice de palatabilidad relativa (IP) se calculó con la ecuación propuesta por Kaitho y col (1996):

\footnotetext{
Valbazen $2,5 \% \circledR$

2 Adechembio®
} 
$\mathrm{IP}=(\mathrm{T} 1 / \mathrm{A} 1) /(\mathrm{T} 1 / \mathrm{A} 1) \mathrm{IP}=(\mathrm{T} 1 / \mathrm{A} 1) /(\mathrm{T} 1 / \mathrm{A} 1)$ para $\mathrm{RM}$.

$\mathrm{IP}=(\mathrm{Ti} / \mathrm{Ai}) /(\mathrm{T} 1 / \mathrm{A} 1)) \mathrm{IP}=(\mathrm{Ti} / \mathrm{Ai}) /(\mathrm{T} 1 / \mathrm{Al})$ para cada fruto.

\section{DISEÑO EXPERIMENTAL}

La composición nutricional de los frutos se analizó en un diseño completamente al azar; modelo estadístico: $\mathrm{Y}_{i j}=\mu+\mathrm{T}_{i}+\xi_{i j}$; donde: $\mathrm{Y}_{i j}=$ variable respuesta (PC, cenizas, MO, FDA, FDN, TT, FT y DMS) al tratamiento ( $i=1,2,3$ frutos) en la repetición $(j=1,2$, 3); $\mu=$ media general; $\mathrm{T}_{i}=$ efecto del tratamiento $(i)$; $\xi_{i j}=$ el error aleatorio del tratamiento $(i)$ en la repetición (j), términos de n-1 $\left(\mathrm{o}^{2}, 0\right)$.

Las variables de la prueba de aceptabilidad se analizaron en un diseño al azar con arreglo factorial de $2 \mathrm{x}$ 3 , que comparó efectos de especie animal, tipo de frutos y sus efectos interactivos; modelo estadístico: $\mathrm{Y}_{i j k}=\mu+$ $\mathrm{A}_{\mathrm{i}}+\mathrm{B}_{j}+(\mathrm{AB})_{i j}+\mathrm{E}_{i j k}$; donde: $\mathrm{Y}_{i j k}=$ variable respuesta (CF e IP) en la repetición $(k=1,2,3)$, nivel $i$ de $(A=1$, 2 especie animal) y nivel $j$ de $(B=1,2,3$ donde: $1=A$. farnesiana, $2=A$. cochliacantha, $3=A$. macilenta); $\mu=$ media general; $\mathrm{A}_{i}=$ efecto del factor $\mathrm{A}$ al nivel $i$, $\mathrm{B}_{j}=$ efecto del factor $\mathrm{B}$ al nivel $j ;(\mathrm{AB})_{i j}=$ efecto de la interacción $\mathrm{A} * \mathrm{~B}$ al nivel $i, j ; \xi_{i j k}=$ error aleatorio, en términos de $\mathrm{n}-1\left(\mathrm{o}^{2}, 0\right)$. Además se realizó un análisis de correlación de Pearson (r) entre la composición química de los frutos, el CF y los IP.

\section{ANÁLISIS ESTADÍSTICO}

Los datos se analizaron por modelos lineales generales (SAS 2001) y la prueba de Tukey $(\mathrm{P}<0,05)$ para comparación de medias.

\section{RESULTADOS}

\section{COMPOSICIÓN QUÍMICA DE LOS FRUTOS}

En el cuadro 1 se observan variaciones $(\mathrm{P}<0,05)$ en la composición química de los frutos. La proteína cruda $(\mathrm{PC})$ y la materia orgánica $(\mathrm{MO})$ fue similar $(\mathrm{P}>0,05)$ entre frutos, el mayor contenido $(\mathrm{P}<0,01)$ de FDN se observó en A. macilenta. El contenido de FDA fue mayor en los frutos de A. macilenta y menor en los de A. cochliacantha $(\mathrm{P}<0,01)$. El mayor contenido de grasa bruta en los frutos de $A$. cochliacantha $(\mathrm{P}<0,05)$ es un indicativo del aporte energético para la dieta de los animales. El contenido de FT y TC fueron mayores $(\mathrm{P}<0,01)$ en los frutos de $A$. farnesiana, comparados a los frutos de A. macilenta y $A$. cochliacantha.

\section{CONSUMO DE MATERIA SECA E ÍNDICE DE PALATABILIDAD}

En el consumo de MS y el IP se observó efecto de la especie animal $(\mathrm{P}<0,01)$ donde las cabras consumieron mayor cantidad de frutos en comparación a las ovejas, sin embargo, el índice de palatabilidad fue mayor en las ovejas (cuadro 2). También se observó efecto de los frutos sobre el consumo de materia seca y el IP que tuvieron los animales (cuadro 2). Los frutos de $A$. cochliacantha y A. farnesiana fueron más consumidos y

Cuadro 1. Composición nutricional de rastrojo de maíz y de frutos de Acacia farnesiana, Acacia cochliacantha, Acacia macilenta $\left(\mathrm{g} \mathrm{kg}^{-1} \mathrm{MS}\right)$.

Nutritional composition of the corn stover and the fruit of Acacia farnesiana, Acacia cochliacantha, Acacia macilenta $\left(\mathrm{g} \mathrm{kg}^{-1} \mathrm{DM}\right)$.

\begin{tabular}{|c|c|c|c|c|c|c|}
\hline & \multirow{2}{*}{$\frac{\text { Dieta base }}{\text { Rastrojo de maíz }}$} & \multicolumn{3}{|c|}{ Frutos } & \multirow{2}{*}{$\mathrm{EE}$} & \multirow{2}{*}{ Significancia } \\
\hline & & A. farnesiana & A. cochliacantha & A. macilenta & & \\
\hline MS & 937,2 & $896,2^{\mathrm{ab}}$ & $885,2^{\mathrm{b}}$ & $910,5^{\mathrm{a}}$ & 2,06 & $*$ \\
\hline MO & 966,1 & 962,6 & 930,7 & 945,3 & 1,59 & ns \\
\hline $\mathrm{PC}$ & 54,0 & 138,1 & 147,3 & 131,6 & 0,76 & ns \\
\hline GB & 9,7 & $7,7^{\mathrm{b}}$ & $12,6^{\mathrm{a}}$ & $9,8^{\mathrm{ab}}$ & 0,12 & $*$ \\
\hline FDN & 741,1 & $537,4^{\mathrm{b}}$ & $550,2^{\mathrm{b}}$ & $635,8^{a}$ & 1,19 & $* *$ \\
\hline FDA & 571,7 & $443,0^{\mathrm{b}}$ & $383,8^{c}$ & $611,3^{\mathrm{a}}$ & 1,28 & $* *$ \\
\hline FT & - & $49,1^{\mathrm{a}}$ & $23,0^{\mathrm{b}}$ & $9,9^{c}$ & 3,25 & $* *$ \\
\hline $\mathrm{TC}$ & - & $9,1^{\mathrm{a}}$ & $3,0^{\mathrm{c}}$ & $3,6^{\mathrm{b}}$ & 0,43 & $* *$ \\
\hline
\end{tabular}

MS: materia seca; MO: materia orgánica; PC: proteína cruda; GB: grasa bruta; FDN: fibra detergente neutro; FDA: fibra detergente ácido; FT: fenoles totales; TC: taninos condensados; EE: error estándar de la media.

a,b,c Valores que difieren en columnas no comparten literal, Tukey $*(\mathrm{P}<0,05) ; * *(\mathrm{P}<0,01)$, ns: no significativo. 
tuvieron mayor IP para las cabras $(\mathrm{P}<0,01)$ y el fruto de A. cochliacantha para las ovejas $(\mathrm{P}<0,05)$. Los efectos de interacción de frutos * especie animal $(\mathrm{P}>0,05)$ solo se observaron en el IP que tuvieron los animales sobre los frutos (cuadro 2).

\section{CORRELACIÓN DE LA COMPOSICIÓN QUÍMICA CON EL CONSUMO DE MATERIA SECA Y LA PALATABILIDAD}

En el cuadro 3 se observa que el contenido de PC de los frutos tuvo correlación alta y positiva $(\mathrm{P}<0,01)$ con el CF y el IP en las ovejas y las cabras. Al contrario, el contenido de fibra detergente ácida tuvo correlación negativa $(\mathrm{P}<0,01)$ con el CF y el IP en las ovejas y las cabras, mientras que el contenido de fibra detergente neutra afectó $(\mathrm{P}<0,01)$ el CF y el IP solo en las cabras (cuadro 3). También el contenido de fenoles totales de los frutos tuvo efecto positivo $(\mathrm{P}<0,05)$ con el $\mathrm{CF}$ y el IP en las ovejas y las cabras sin embargo la correlación fue mayor en las cabras en comparación a las ovejas (cuadro 3). Los taninos condensados y el contenido de grasa cruda en los frutos no fueron determinantes en el consumo de frutos y el índice de preferencia observado en los animales (cuadro 3).

Cuadro 2. Consumo de materia seca (MS) e índice relativo de palatabilidad (IP) de frutos en ovejas y cabras. Dry matter intake (DM) and relative palatability index (PI) of fruit in sheep and goats.

\begin{tabular}{|c|c|c|c|}
\hline Especie animal & Alimento & Consumo MS (g/día) & Índice de palatabilidad \\
\hline \multirow[t]{5}{*}{ Ovejas } & Rastrojo de maíz & $100,00^{\mathrm{d}}$ & $1,00^{\mathrm{c}}$ \\
\hline & A. farnesiana & $284,58^{b}$ & $2,85^{\mathrm{b}}$ \\
\hline & A. cochliacantha & $342,18^{a}$ & $3,42^{\mathrm{a}}$ \\
\hline & A. macilenta & $142,53^{\mathrm{cd}}$ & $1,43^{\mathrm{c}}$ \\
\hline & Promedio de frutos & $256,43^{\mathrm{i}}$ & $2,57^{\mathrm{ii}}$ \\
\hline \multirow[t]{8}{*}{ Cabras } & Rastrojo de maíz & $142,18^{\mathrm{cd}}$ & $1,00^{\mathrm{c}}$ \\
\hline & A. farnesiana & $352,28^{\mathrm{a}}$ & $2,48^{\mathrm{b}}$ \\
\hline & A. cochliacantha & $358,64^{\mathrm{a}}$ & $2,52^{\mathrm{b}}$ \\
\hline & A. macilenta & $170,99^{\mathrm{cd}}$ & $1,20^{\mathrm{c}}$ \\
\hline & Promedio de frutos & $293,97^{\mathrm{ii}}$ & $2,08^{\mathrm{i}}$ \\
\hline & Frutos & $* *$ & $* *$ \\
\hline & Especie animal & $*$ & $* *$ \\
\hline & Frutos * Especie animal & ns & $*$ \\
\hline
\end{tabular}

a,b,c Diferentes literales en la misma columna indican diferencias: $*(\mathrm{P}<0,05) ; * *(\mathrm{P}<0,01)$, ns: no significativo.

i,iivalores promedio diferentes por efecto de la especie animal.

Cuadro 3. Correlación de la composición nutricional de los frutos de acacias con el consumo de materia seca y el índice de palatabilidad relativa en ovejas y cabras.

Correlation between nutritional composition of fruits of acacias and dry matter intake and relative palatability index sheep.

\begin{tabular}{|c|c|c|c|c|}
\hline \multirow{2}{*}{ Compuesto químico } & \multicolumn{2}{|c|}{ Ovejas (r) } & \multicolumn{2}{|c|}{ Cabras (r) } \\
\hline & $\mathrm{CF}$ & IP & $\mathrm{CF}$ & IP \\
\hline Proteína cruda & $0,83 * *$ & $0,83 * *$ & $0,79 * *$ & $0,79 * *$ \\
\hline Grasa bruta & $0,32 \mathrm{~ns}$ & $0,31 \mathrm{~ns}$ & $0,11 \mathrm{~ns}$ & $0,11 \mathrm{~ns}$ \\
\hline Fibra detergente neutro & $-0,38 \mathrm{~ns}$ & $-0,38 \mathrm{~ns}$ & $-0,61 * *$ & $-0,61 * *$ \\
\hline Fibra detergente ácido & $-0,61 * *$ & $-0,62 * *$ & $-0,82 * *$ & $-0,81 * *$ \\
\hline Fenoles totales & $0,48 *$ & $0,48 *$ & $0,71 *$ & $0,71 *$ \\
\hline Taninos condensados & $0,13 \mathrm{~ns}$ & $0,13 \mathrm{~ns}$ & $0,38 \mathrm{~ns}$ & $0,38 \mathrm{~ns}$ \\
\hline
\end{tabular}

CF: Consumo de frutos, IP: Índice de palatabilidad relativa.

Significancia, Tukey $*(\mathrm{P}<0,05), * *(\mathrm{P}<0,01)$, ns: no significativo. 


\section{DISCUSIÓN}

\section{COMPOSICIÓN QUÍMICA DE LOS FRUTOS}

El contenido de $\mathrm{PC}$ de los frutos evaluados es mayor al de gramíneas en estado maduro, esto indica la importancia de los frutos en el aporte de este nutriente a la dieta de pequeños rumiantes (García y col 2011). Además, el contenido de PC, FDN y FDA en los frutos de las acacias es comparable al reportado por otros investigadores (Smith y col 2005, Olivares y col $2013^{b}$ ). El aporte de proteína de los tres frutos resulta apropiado para aportar el 8,0\% de nitrógeno que requiere la flora microbiana del rumen para un adecuado funcionamiento durante la digestión del alimento (Norton 1998).

\section{CONSUMO DE MATERIA SECA E ÍNDICE DE PALATABILIDAD}

El contenido de taninos condensados y fenoles totales en los frutos fue bajo, lo que originó que no se afectara el CF y el IP en las ovejas y las cabras (cuadros 1 y 2). Min y col (2003), Mahgoub y col (2005), Mueller (2006) y Alonso y col (2009), reportaron que alimentos con concentraciones de FT y TC que no superaron los $50 \mathrm{~g} / \mathrm{kg}$ de MS, no causaron intoxicaciones ni tuvieron efecto en la disminución del consumo de materia seca por los animales.

En la comparación de la especie animal (cuadro 2), las cabras consumieron más frutos que las ovejas debido a diferencias fisiológicas que demuestran que las cabras toleran mayor consumo de compuestos astringentes como los taninos debido a la secreción de saliva rica en prolina, enzima que neutraliza el efecto de los taninos (Osuga y col 2008, Alonso y col 2009).

El alto consumo y el IP sobre los frutos de $A$. cochliacantha y A. farnesiana por las cabras y ovejas (cuadro 2) se atribuyó a que son especies forrajeras que tienen alta densidad en los trópicos (Olivares y col 2011) y por lo tanto los animales han tenido una adaptación natural a consumir sus frutos (Torres y col 2008, Goetsch y col 2010). Además, el mayor contenido de $\mathrm{PC}$ y el menor contenido de fibras detergentes en frutos de A. cochliacantha y $A$. farnesiana (cuadro 1) estimularon el consumo de materia seca y el IP en las ovejas y las cabras (cuadro 2) similar a lo reportado por Olivares y col $\left(2013^{\mathrm{b}}\right)$. Lu y col (2008) observaron que alimentos que superaron el $41 \%$ en el contenido de FDN disminuyeron el consumo de materia seca en cabras. Este comportamiento indica que la preferencia que tuvieron las cabras y las ovejas sobre un alimento está determinada por las propiedades organolépticas debidas a la composición química del alimento y a características inherentes al animal.

El efecto interactivo frutos*especie animal observado en el IP (cuadro 2) se atribuye al ajuste que se realizó con el consumo del rastrojo de maíz dentro de la especie animal. En general el consumo y el IP observado en las cabras y ovejas sobre los frutos de A. farnesiana y A. cochliacantha fue mayor comparado a los reportados por Smith y col (2005), Mokoboki y col (2011) y Yinnesu y Nurfeta (2012), lo que demuestra el potencial alimenticio de estas estructuras para la alimentación de pequeños rumiantes en el trópico.

\section{CORRELACIÓN DE LA COMPOSICIÓN QUÍMICA CON EL CONSUMO DE MATERIA SECA Y LA PALATABILIDAD}

En el análisis de correlación se observó en las cabras y las ovejas que cuando consumen frutos con bajo contenido de compuestos secundarios (FT y TC) como los utilizados en este estudio (cuadro 3), el consumo de materia seca y la palatabilidad es regulado por otros nutrientes como la PC, FDN y FDA, resultados que fueron similares a los reportados por Mueller (2006), Alonso y col (2009), Mokoboki (2011) y Olivares y col $\left(2013^{a}\right)$.

La correlación positiva observada en el consumo de materia seca y el IP en las ovejas y cabras (cuadro 3) indica que los animales prefirieron consumir los frutos de A. cochliacanta y A. farnesiana por su mayor contenido proteico (cuadro 1), comparados con el fruto de A. macilenta. Esta conducta es similar a la reportada por Mokoboki y col (2011) y Olivares y col $\left(2013^{\mathrm{a}}\right.$ y $\left.2013^{\mathrm{b}}\right)$. El análisis de correlación también indica que la conducta de las ovejas y las cabras al consumo y la palatabilidad de los frutos de A. cochliacanta y A. farnesiana se atribuyó al menor contenido de FDA y FDN, comparado con el fruto de A. macilenta (cuadros 1 y 2). Estas variaciones se atribuyen a las diferencias en la lignificación de las paredes celulares de los frutos otorgadas por la especie arbórea (Abdallah y col 2010) y no a la fenología de los frutos, debido a que fueron cosechados en el mismo período, área geográfica y en etapa de madurez de los frutos. Los procesos de lignificación aumentan el contenido de fibras detergentes en los alimentos y estas a su vez afectan la digestibilidad y el consumo de materia seca por los animales (Njidda and Ikhimioya 2010, Rojas y col 2013).

Se concluye que el consumo de los frutos y el índice de preferencia por las ovejas y las cabras estuvieron determinados por el contenido de proteína cruda, fibra detergente neutra y fibra detergente ácida. La proteína cruda y el menor contenido de fibras detergentes en los frutos de A. cochliacanta y A. farnesiana dan mayor potencial alimenticio para ser utilizados como alimento en las ovejas y las cabras, en comparación con los frutos de A. macilenta. El bajo contenido de FT y TC en los frutos no afectó el consumo de materia seca por las ovejas y las cabras, sin embargo las cabras por sus hábitos alimenticios consumieron más fruto que las ovejas. El contenido de fibras detergentes en los frutos es la principal limitante que puede afectar el consumo de materia seca en pequeños rumiantes. 


\section{REFERENCIAS}

Abdallah HSSM, BIC da Silva, GP Barbosa, EF Nozella, VDMS Schmidt, AL Abdalla. 2010. Ruminal fermentation and tannins bioactvity of some browses ising a semi-automated gas production technique. Trop Subtrop Agroecosyst 12, 1-10.

Alonso MA, JFJ Torres, C Sandoval, HL Canul, H Hoste. 2009. Intake of tropical tanniniferous plants by goats and sheep when offered as a sole fed. Trop Subtrop Agroecosyst 11, 255-258.

Álvarez G, L Melgarejo, Y Castañeda. 2003. Ganancia de peso, conversión y eficiencia alimentaria en ovinos alimentados con fruto (semilla con vaina) de parota (Enterolobium cyclocarpum) y pollinaza. Vet Méx 34, 39-46.

Association of Official Analytical Chemists. 2000. Official methods of analysis. $17^{\text {th }}$ ed. AOAC, Arlington, VA, USA.

Dey A, N Dutta, K Sharma, AK Pattanaik. 2008. Effect of dietary inclusion of Ficus infectoria leaves as a protectant of proteins on the performance of lambs. Small Rumin Res 75, 105-114.

Fragoso C. 1990. Monografía del estado de Guerrero, sur amate de mar y montaña. SEP, México, D.F.

García LR, S Goñi, PA Olguín, G Díaz, CM Arriaga. 2009. Huizache (Acacia farnesiana) whole pods (flesh and seeds) as an alternative feed for sheep in Mexico. Trop Anim Health Prod 41, 1615-1621.

García CA, M González, AZM Salem, J Romero, JF Pedraza, JG Estrada. 2011. Chemical composition and in vitro gas production of some legume browse species in subtropical areas of Mexico. Short note. Trop Subtrop Agroecosyst 14, 589-595.

Goetsch AL, TA Gipson, AR Askar, R Puchala. 2010. Feeding behavior of goats. J Anim Sci 88, 361-373.

Kaitho RJ, NN Umunna, IV Nsahlai, S Tamminga, J Van Bruchem, J Hanson, M Van de Wouw. 1996. Palatability of multipurpose tree species: effect of species and length of study on intake and relative palatability by sheep. Agrofor Syst 13, 249-261.

Kalio GA, UI Oji, A Larbi. 2006. Preference and palatability of indigenous and exotic acid soil-tolerant multipurpose trees and shrubs byWest African Dwarf sheep. Agrofor Syst 67, 123-128.

Khan M, F Hussain. 2012. Palatability and animal preferences of plants in Tehsil Takht-e-Nasrati, District Karak, Pakistan. Afr J Agric Res 7, 5858-5872.

Lu DC, RJ Kawas, GO Mahgoub. 2008. Recent advancements in fiber digestion and utilization in goats. Trop Subtrop Agroecosyst 9, 65-72.

Mahgoub O, IT Kadim, NE Forsberg, DS Al-Ajmi, NM Al-Saqry, AS AlAbri, K Annamalai. 2005. Evaluation of Meskit (Prosopis juliflora) pods as a feed for goats. Anim Feed Sci Techn 121, 319-327.

Min BR, TN Barry, GT Attwood, WC McNabb. 2003. The effect of condensed tannins on the nutrition and health of ruminants fed fresh temperate forages. Anim Feed Sci Technol 106, 3-19.

Mlambo V, FL Mould, JLN Sikosana, T Smith, E Owen, I Mueller. 2008. Chemical composition and in vitro fermentation of tannin-rich tree fruits. Anim Feed Sci Technol 140, 402-417.

Mokoboki HK, LR Ndlovu, MM Malatje. 2011. Intake and relative palatability indices of acacia species fed to sheep and goats. Agrofor Syst 81, 31-35.

Mueller I. 2006. Unravelling the conundrum of tannins in animal nutrition and health. $J$ Sci Food Agric 86, 2010-2037.

Ngwa AT, IV Nsahlai, MLK Bonsi. 2003. Feed intake and dietary preferences of sheep and goats offered hay and legume-tree pods in South Africa. Agrofor Syst 57, 29-37.

Njidda AA, I Ikhimioya. 2010. Correlation between chemical composition and in vitro dry matter digestibility of leaves of semi-arid browses of North-Eastern, Nigeria. Am Eurasian J Agric Environ Sci 9 , 169-175.

Norton BW. 1998. The nutritive value of tree legumes. In: Gutteridge RC, Shelton HM (eds). Forage tree legumes in tropical agriculture. The Tropical Grassland Society of Australia Inc., Brisbane, Australia, Pp 202-215.

Olivares J, F Avilés, B Albarrán, S Rojas, OA Castelán. 2011. Identificación, usos y medición de leguminosas arbóreas forrajeras en ranchos ganaderos del sur del estado de México. Trop Subtrop Agroecosyst 14, 739-748.

Olivares PJ, NF Avilés, PB Albarrán, OOA Castelán, HS Rojas, 2013․ Use of three fodder trees in the feeding of goats in the subhumid tropics in Mexico. Trop Anim Health Prod 45, 821-828.

Olivares J, F Avilés, B Albarrán, OA Castelán, S Rojas. 2013 . Nutritional quality of Pithecellobium dulce and Acacia cochliacantha fruits, and its evaluation in goats. Livest Sci 154, 74-81.

Osuga IM, CC Wambui, SA Abdulrazak, T Ichinohe, T Fujihara. 2008. Evaluation of nutritive value and palatability by goats and sheep of selected browse foliages from semiarid area of Kenya. Anim Sci $J 79,582-589$.

Rahim I, JI Sultan, M Sharif, MQ Bilal. 2013. Chemical composition, mineral profile, palatabiltiy and in vitro digestibility of shrubs. $J$ Anim Plant Sci 23, 45-49.

Rodríguez R, M Fondevila, C Castrillo. 2009. In vitro fermentation of Pennisetum purpureum CT- 115 supplemented with four tropical browse legume species. Anim Feed Sci Technol 151, 65-74.

Rojas S, F Avilés, O Castelán, A García, J Olivares, MT Valencia. 2012. Chemical Composition, in vitro digestibility of foliage Guazuma ulmifolia and Crescentia alata and its use in feeding lambs. Pak $J$ Nutr 11, 1139-1145.

Rojas-Hernández S, J Olivares-Pérez, I Gutiérrez-Segura, R JiménezGuillén, F León-López, A Córdova-Izquierdo. 2013. Use of Crescentia alata and Guazuma ulmifolia fruits in lamb feeding in subtropical region of Guerrero, Mexico. Rev Científ FCV-LUZ 23, 157-162.

SAS. 2001. SAS/StAt User's Guide, Version 8.2. SAS Institute Inc, Cary NC, USA.

Smith T, V Mlambo, JLN Sikosana, V Maphosa, I Mueller, E Owen. 2005. Dichrostachys cinerea and Acacia nilotica fruits as dry season feed supplements for goats in a semi-arid environment: Summary of a DFID funded project in Zimbabwe. Anim Feed Sci Technol $122,149-157$

Sosa EE, D Pérez, L Ortega, G Zapata. 2004. Evaluación del potencial forrajero de árboles y arbustos tropicales para la alimentación de ovinos. Tec Pec Méx 42, 129-144.

Torres AJFJ, DMA Alonso, H Hoste, CCA Sandoval, CAJ Aguilar. 2008. Positive and negative effects in goat production arising from the intake of tannin rich forage. Trop Subtrop Agroecosyst 9, 83-90.

Van Soest PJ, J Robertson, B Lewis. 1991. Methods for dietary fiber, neutral detergent fiber and nonstarch polysaccharides in relation to animal nutrition. J Dairy Sci 74, 3583-3595.

Vázquez MP, O Castelán, A García, F Avilés. 2012. Uso de bloques nutricionales como complemento para ovinos en el trópico seco del altiplano central de México. Trop Subtrop Agroecosyst 15, 87-96.

Waterman PG, S Mole. 1994. Analysis of phenolic plant metabolites. Blackwell Scientific Publications, London, UK.

Yinnesu A, A Nurfeta. 2012. Effects of supplementing Erythrina brucei leaf as a substitute for cotton seed meal on growth performance and carcass characteristics of Sidama goats fed basal diet of natural grass hay. Trop Anim Health Prod 44, 445-451. 